\title{
NOVOS TRATAMENTOS PARA O DIABETES MELLITUS TIPO 2
}

\author{
NEW TREATMENTS FOR TYPE 2 DIABETES MELLITUS
}

\author{
Andressa Moraes Mota Freitas ${ }^{1}$, Bianca Moreira Belido1, Raphael Azevedo Rodrigues da Silva', Charbell Miguel \\ Haddad Kury $2,3,4,5$. \\ 1Acadêmico da Faculdade de Medicina de Campos. \\ 2Professor Titular de Bioquímica da Faculdade de Medicina de Campos. \\ 3Doutor e Mestre em Microbiologia e Parasitologia Aplicada. \\ 4Membro do Departamento de Infectologia da SOPERJ. \\ 5Título de Especialista em Pediatria, Infectologia Pediátrica e Neonatologia pela SBP.
}

\section{RESUMO}

Introdução: O diabetes mellitus tipo 2 é uma doença crônica que apresenta níveis elevados de glicemia e que pode acarretar complicações, havendo necessidade da mudança do hábito de vida e da utilização de medicações. Métodos: Trata-se de um estudo do tipo revisão de literatura, para o qual foi realizado um levantamento de estudos sobre novos tratamentos do diabetes mellitus do tipo 2 nas bases de dados Google Acadêmico, Scientific Electronic Library Online (Scielo), PubMed e órgãos de importância na área como a Sociedade Brasileira de Diabetes e Sociedade Brasileira de Endocrinologia e Metabologia. Discussão: Primariamente, o paciente diabético deve iniciar um tratamento não medicamentoso com uma alimentação balanceada e prática de atividade física. Após essa mudança, caso necessário, são adicionados medicamentos para auxiliar no controle da doença, como metformina, sulfonilureias, tiazolinedionas entre outros. Esses medicamentos podem ser prescritos em monoterapia ou associados a outros hipoglicemiantes. Caso a doença não seja controlada com esses medicamentos, pode-se incluir a suplementação de insulina ou optar pelos novos tratamentos disponíveis. Conclusão: Ao abordar os tratamentos para o diabetes mellitus tipo 2, enfatizando as novas opções terapêuticas, como os agonistas dos receptores da GLP-1, inibidores da DPP-4 e os inibidores de SGLT-2, observa-se a importância de estudar novos

\section{ABSTRACT}

Introduction: Type 2 diabetes mellitus is a chronic disease that presents high blood glucose levels and can lead to complications, requiring a change in lifestyle and the use of medications. Objectives: This is a literature review study, for which a survey of studies on new treatments of type 2 diabetes mellitus was carried out in the Google Scholar, Scientific Electronic Library Online (Scielo), PubMed and important organs databases in the area like the Brazilian Society of Diabetes and the Brazilian Society of Endocrinology and Metabology. Methods: Primarily, the diabetic patient should start a non-medication treatment with a balanced diet and physical activity. After this change, if necessary, drugs are added to help control the disease, such as metformin, sulfonylureas and thiazolinediones. These drugs can be prescribed as monotherapy or associated with other hypoglycemic agents. If the disease is not controlled with these drugs, you can include insulin supplementation or opt for the new treatments available. Conclusion: When addressing treatments for type 2 diabetes mellitus, emphasizing new therapeutic options, such as GLP-1 receptor agonists, DPP-4 inhibitors and SGLT-2 inhibitors, it is the observable the importance of studying new drugs that in addition to regulating blood glucose, it can bring other benefits such as weight loss and cardiorenal protection, depending on the medication. In this review, we emphasize the importance of changing lifestyle, in addition to adherence to drug treatment so that the damages 
fármacos que além de regularem a glicemia, podem trazer outros benefícios como perda de peso e proteção cardiorrenal, a depender da medicação. Nesta revisão, ressaltamos a importância da mudança do estilo de vida, além da adesão ao tratamento medicamentoso para que os danos causados pela descompensação da doença não existam ou sejam minimizados.

PALAVRAS-CHAVE: diabetes mellitus tipo 2, tratamento, agonistas dos receptores da GLP-1, inibidores da DPP-4, inibidores da SGLT-2

\section{INTRODUÇÃO}

O diabetes mellitus se apresenta como uma síndrome metabólica que tem como característica níveis elevados de glicose na corrente sanguínea ocasionados pela resistência à ação da insulina ou até mesmo pela diminuição ou deficiência da secreção da mesma'. A insulina é um hormônio produzido pelas células beta pancreáticas das ilhotas de Langerhans que, inicialmente, produzem uma maior quantidade deste hormônio para que haja o controle glicêmico, mas, no decorrer da manifestação da doença, estas células ficam sobrecarregadas e, consequentemente danificadas, reduzindo a sua capacidade de produção, o que gera um quadro de resistência periférica à insulina, onde há uma menor captação de glicose pelos tecidos e aumento da liberação de glicose pelo fígado. Este hormônio é secretado após período prandial, ou seja, o indivíduo precisa estar alimentado'. Esta doença é classificada em 2 tipos: diabetes tipo 1, cuja característica se dá pela deficiência de secreção da insulina e geralmente se apresenta em crianças, adolescentes e adultos jovens; enquanto o diabetes tipo 2 é mais prevalente em pessoas adultas maiores de 40 anos, pela diminuição da secreção de insulina, resistência à sua ação e, em casos mais graves, pela deficiência da secreção deste hormônio. A maioria dos casos de diabetes é do tipo 2 devido ao aumento da obesidade na população, maus hábitos alimentares, sedentarismo e também ao envelhecimento populacional'.

Esta doença apresenta uma prevalência de $8,3 \%$ na população mundial e, no Brasil são mais de 12 milhões de doentes e, segundo a International Diabetes Federation (IDF), um em cada dois indivíduos acometidos pelo diabetes não sabem que caused by decompensating the disease do not exist or are minimized.

KEYWORDS: type 2 diabetes mellitus, treatment, GLP-1 receptor agonists, DPP-4 inhibitors, SGLT-2 inhibitors

estão doentes, ou seja, 50\%. O diabetes mellitus tipo 2 é uma enfermidade que se tornou um problema de saúde pública mundial e, até 2035 , estima-se que 592 milhões de pessoas serão afetadas por esta doença crônica'.

Quando não há adesão ao tratamento, podem ocorrer complicações que comprometem a qualidade de vida podendo levar à morte. Essas complicações podem ter origem na microvascularização, causando retinopatia e nefropatia, na macrocirculação, levando a cardiopatia isquêmica, doença cerebrovascular e doença vascular periférica e até mesmo pode ser de origem neuropática ${ }^{2}$.

O tratamento do diabetes mellitus tipo 2 consiste principalmente em promover o controle glicêmico ideal, utilizando como estratégias tanto uma dieta hipocalórica associada à atividade física quanto ao uso de medicações. Em relação às medicações utilizadas no tratamento do diabetes mellitus tipo 2, temos as insulinas de liberação lenta como a NPH, de liberação ultra lenta como a insulina Glargina e Degluteca; e as insulinas rápidas e ultra rápidas como a regular, LisPro e Glulisina; os agentes hipoglicemiantes orais como metformina, tiazolinodinodionas, secretagogos, como as sulfonilureias e as novas terapias, como os inibidores do cotransportador sódio-glicose 2 (SGLT-2) e os incretinomiméticos, que são os agonistas dos receptores de GLP1 e os inibidores de dipeptidilpeptidase-4 (DPP-4) ${ }^{1}$.

\section{OBJETIVOS}

Este trabalho teve como objetivo fazer uma revisão de literatura sobre as novas opções terapêuticas no tratamento do diabetes mellitus 
tipo 2.

\section{MÉTODOS}

Trata-se de um estudo do tipo revisão de literatura, para o qual foi realizado um levantamento de estudos sobre novos tratamentos do diabetes mellitus do tipo 2. As palavras-chave utilizadas para a busca de resultados deste estudo foram: tratamento, hipoglicemiantes orais, inibidores da DPP-4, agonistas dos receptores da GLP-1, inibidores da SGLT-2, diabetes mellitus e diabetes tipo 2. Como critérios de inclusão, foram selecionados artigos científicos em língua portuguesa, publicados entre os anos de 2015 a 2020, nas bases de dados Google Acadêmico, Scientific Electronic Library Online (Scielo), PubMed e órgãos de importância na área como a Sociedade Brasileira de Diabetes e Sociedade Brasileira de Endocrinologia e Metabologia. Foram excluídos os artigos publicados antes de 2015.

\section{TÓPICOS A SEREM ABORDADOS}

$O$ diabetes mellitus é uma doença crônica caracterizada pela hiperglicemia, que pode ser ocasionada por falhas na ação e/ou na secreção de insulina. ${ }^{3} \mathrm{O}$ tratamento do diabetes mellitus tipo 2 tem como objetivo manter o paciente livre de sinais e sintomas relacionados à hiperglicemia e, consequentemente, impedir as complicações agudas e diminuir ou evitar as complicações crônicas da doença, bem como deixar o paciente apto a realizar atividades física, mental, laboral e social com melhor qualidade de vida. ${ }^{4}$

Para que o tratamento tenha um controle satisfatório, são necessários alguns fatores que condicionam o diabetes mellitus tipo 2 para uma glicemia adequada, como uma dieta balanceada, atividade física e adesão ao tratamento; assim como é necessário que diminua o estresse e evite infecções. ${ }^{4}$

Dentre os medicamentos utilizados para o tratamento do diabetes mellitus tipo 2, temos:

\section{Sulfonilureias}

As sulfonilureias estimulam as células $\beta$ pancreáticas a secretar mais insulina pela inibição do canal de $\mathrm{K}+$ sensível ao ATP, que provoca uma despolarização e ativa os canais de $\mathrm{Ca}++$ levando a uma exocitose da insulina armazenada e aumenta a concentração plasmática da mesma. ${ }^{4}$
Dentre as reações adversas, as principais são o ganho de peso e hipoglicemia, devido a uma maior meia vida plasmática. As sulfonilureias são contraindicadas na gestação e no diabetes mellitus tipo 1 , pois nesse tipo de diabetes as células $\beta$ não são funcionais. ${ }^{4}$

\section{Meglitinidas}

As meglitinidas possuem o mesmo mecanismo de ação das sulfonilureias, mas estimulam a secreção prandial de insulina, ou seja, na presença de alimento. Por possuir uma meia-vida menor que das sulfonilureias, o risco de hipoglicemia é diminuído e são contraindicadas principalmente na gestação, lactação e insuficiência renal e hepática severas.

\section{Biguanidas}

As biguanidas (metformina) melhora a captação da glicose pelas células, melhorando a afinidade entre a insulina e seu receptor pelo aumento da atividade da proteinocinase dependente de AMP (AMPK). Ao ativar a AMPK, a gliconeogênese é reduzida e há aumento da sensibilidade à insulina e do armazenamento de glicogênio no músculo esquelético. ${ }^{4}$

Seus efeitos adversos mais comuns são efeitos gastrintestinais, como diarreia e redução na absorção de vitamina B12. A metformina é contraindicada na doença renal ou hepática, gravidez, insuficiência cardíaca, gravidez. ${ }^{4}$

\section{Tiazolidinedionas}

As tiazolinedionas (rosiglitazona e pioglitazona) atuam na redução da resistência à insulina, no aumento do transporte de glicose e ainda tem efeito na melhora do perfil lipídico. 4

Ganho de peso, edema e efeitos cardiovasculares são seus efeitos adversos mais comuns, além disso, são contraindicadas em pacientes com doenças hepáticas e com insuficiência cardíaca. ${ }^{4}$

\section{Inibidores da $\alpha$ glicosidade}

Os medicamentos inibidores da $\alpha$ glicosidase retardam a absorção de glicose, reduzindo a glicemia pós-prandial São exemplos dessa classe acarbose e miglitol. ${ }^{4}$

Pacientes com distúrbios intestinais, insuficiência cardíaca, insuficiência hepática e 
mulheres grávidas não devem utilizar essa classe de medicamentos. Os efeitos adversos mais comuns são flatulência, diarreia, distensão abdominal, aumento do peso, edema e aumento das enzimas hepáticas.

\section{Insulina}

A insulinoterapia é realizada no paciente diabético tipo 2 num estágio avançado da doença, onde as células $\beta$ pancreáticas já estão muito comprometidas, ou seja, não conseguem produzir e secretar insulina o suficiente. Nesse caso, o tratamento insulínico se faz necessário.

No início do tratamento com a insulina, é utilizada uma insulina intermediária, que mantém um nível basal da mesma, associando com o uso de hipoglicemiantes orais. Caso haja uma hiperglicemia pós-prandial não controlada, é necessário que se acrescente o uso da insulina prandial, de ação curta ou ação rápida. ${ }^{5}$

A insulina de ação intermediária (basal) é a humana NPH e tem uma duração mais prolongada. Já as insulinas de ação curta (humana regular) e rápida (lispro, asparte e glulisina) tem um efeito mais imediato, sendo utilizadas antes das refeições para manter a glicemia em níveis adequados. ${ }^{5}$

\section{Novas opções}

Com o avanço da ciência, surgiram novas medicações para o tratamento do diabetes mellitus tipo 2, que são os incretinomiméticos (agonistas dos receptores da GLP-1 e inibidores da DPP-4) e os inibidores dos cotransportadores de sódio glicose 2 (inibidores da SGLT-2).

As incretinas conhecidas como GLP-1 e GIP, são os únicos substratos da protease DPP-4 que foram eventualmente reconhecidos nos seres humanos. ${ }^{6}$

Sabe-se que o GLP-1 (glucagon-like peptide 1) é um hormônio incretínico liberado após as refeições, secretado pelo intestino delgado e que funciona de modo a aumentar a secreção de insulina e diminuir a liberação do glucagon. Além disso, o GLP-1 atua regulando o apetite e saciedade, sendo responsável por retardar o esvaziamento gástrico. ${ }^{7},{ }^{8}$

A produção de GLP-1 é diminuída em pessoas que apresentam diabetes mellitus do tipo 2 , uma vez que o GLP-1 é produzido e rapidamente degradado pela enzima DPP-4, portanto tem uma meia-vida plasmática em torno de 2 minutos. ${ }^{8,}$,

\section{Agonistas dos receptores da GLP-1}

Os fármacos agonistas dos receptores da GLP1 são homólogos ao GLP-1 humano, possuem administração subcutânea e tem maior eficácia no controle diabético. ${ }^{7}$ Atuam mimetizando os efeitos do GLP-16, de forma que devido a um aumento nas concentrações plasmáticas de glicose, os agonistas dos receptores da GLP-1 atuam nas células $\beta$ pancreáticas estimulando a secreção de insulina ao mesmo tempo que atuam nas células $\alpha$ pancreáticas inibindo a secreção de glucagon. Isso acontece devido aos receptores da GLP-1 presentes nas membranas das células pancreáticas que permitem a ligação desses fármacos, assim como estão presentes também nos pulmões, coração, vasos sanguíneos, trato gastrintestinal, rins, mama e Sistema Nervoso Central. Isso nos leva aos seus efeitos extra pancreáticos, como por exemplo, o atraso no esvaziamento gástrico e na promoção da saciedade. $^{9}$

Além desses efeitos, os agonistas dos receptores da GLP-1 promovem efeitos cardiovasculares, como redução da pressão arterial sistólica, bem como efeitos protetores no miocárdio, na hipertensão, na aterosclerose, melhora no perfil lipídico, o que também está associado à diminuição do peso, porém esses mecanismos ainda não estão completamente elucidados. ${ }^{9}$

No Brasil, temos exenatida e liraglutida de uso diário e dulaglutida e semaglutida de uso semanal como agonistas dos receptores da GLP-1, que podem ser associados com outros antidiabéticos orais ou insulina, sendo o primeiro com uma homologia de cerca de 50\% com o GLP-1 humano; enquanto os demais chegam a $97 \%$ de homologia. ${ }^{9}$

Os agonistas dos receptores da GLP-1 possuem efeitos satisfatórios no controle glicêmico, perda de peso e na redução da pressão arterial. Reduzem a hemoglobina glicada (HbA1c) média em 0,55-1,2\% em comparação ao placebo em pacientes com diabetes mellitus tipo 2 , reduz a pressão arterial sistólica e diastólica em cerca de 1,2-4,6 mm Hg sistólica / 0-1,1 mmHg diastólica, sendo esses efeitos independentes da perda de peso e do controle glicêmico, pois estão associados ao aumento da frequência cardíaca. Quanto à perda de peso, varia de $0,2-7,2 \mathrm{~kg}$, com aproximadamente $50 \%$ dos pacientes atingindo perda de $5 \%$ do peso durante 0 tratamento. ${ }^{10}$

Há estudos que mostram que esses fármacos 
ainda podem apresentar efeito nefroprotetor, o que pode representar formas de prevenção ou melhoria da nefropatia diabética, estimulando a excreção renal de sódio e diminuindo citocinas inflamatórias e fatores profibróticos. Porém, também foram referidos alguns casos de lesão renal aguda.. ${ }^{7}$ Quanto aos efeitos adversos, os agonistas dos receptores da GLP-1 estão principalmente associados ao trato gastrintestinal, como náuseas, vômitos e diarreia, mas que que são rápidos ou moderados e de caráter provisório. ${ }^{9},{ }^{10}$

A liraglutida tem maior eficácia na perda de peso e é o único agonista dos receptores da GLP-1 que tem evidência científica de reduzir o risco cardiovascular em doentes com diabetes mellitus tipo 2 e risco cardiovascular elevado, além disso também diminui a mortalidade. Os pacientes que mais se beneficiam são os que possuem mais de 50 anos, com doença cardiovascular estabelecida, IMC maior que $30 \mathrm{~kg} / \mathrm{m} 2$ e taxa de filtração glomerular menor que $60 \mathrm{ml} / \mathrm{min} / 1,73 \mathrm{~m} 2$. Outros benefícios desse fármaco são a diminuição da HbA1c de 0,7 a $1,8 \%$ na dose de $1,8 \mathrm{mg}$ em comparação com outros fármacos e também a diminuição do perímetro abdominal e gordura visceral. ${ }^{9}$

\section{Inibidores de DPP-4}

A dipeptidil peptidase-4 conhecida como DPP-4 é tida como uma protease da serina formada por 766 aminoácidos. É uma enzima que se encontra amplamente distribuída em várias partes distintas do corpo. Como exemplo podemos citar os rins, fígado, baço, placenta, glândulas suprarrenais, intestinos, células endoteliais. ${ }^{6}$

Como já foi enunciado a DPP-4 degrada e inativa as incretinas GLP-1 e GIP, sendo que com a GLP-1 isso ocorre em poucos minutos (2,4 minutos), a inibição da DPP-4 é uma outra alternativa terapêutica no tratamento do diabetes, uma vez que a inibição desta enzima, tem como consequência o aumento dos níveis circulantes de GLP-1 ativos. ${ }^{6}$

Inibidores da dipeptidil peptidase 4 (IDPP4), também conhecidos como gliptinas, são considerados uma nova classe de hipoglicemiantes orais utilizados no tratamento do diabetes mellitus tipo 2. O primeiro inibidor de DPP-4 foi aprovado pelo FDA em 2006, intitulados de sitagliptina e vildagliptina, pertencem à classe dos inibidores da DPP-4 que estão sendo utilizados em diversos países pelo mundo. Alogliptina, saxagliptina e linagliptina são inibidores que também já estão sendo comercializados em diversas nações, e muitos outros ainda aguardam aprovação. Podem ser combinados com outros fármacos antidiabéticos, usualmente com metformina, no mesmo comprimido. ${ }^{6}, 11$

Em relação aos inibidores de DPP-4, os estudos clínicos mostraram em seu resultado uma eficácia não inferior aos antidiabéticos mais utilizados e uma boa tolerância com uma quase ausência de efeitos adversos ou de episódios de hipoglicemia com efeito nulo e ainda se mostrou um pouco benéfico no peso corporal. Uma das características que facilitaram muito sua adesão é ao fato de sua administração ser oral e em doses fixas sem a necessidade de se escalonar. ${ }^{11}$

Os iDPP-4 não são considerados como a terapia inicial para o tratamento do diabetes mellitus tipo 2, na maioria dos pacientes a terapia inicial deve começar com dieta especifica para diabetes, redução de peso, exercícios e metformina. Os inibidores da DPP-4 podem ser utilizados como terapia adjuvante medicamentosa associados com outros fármacos antidiabéticos, utilizado principalmente em combinação com a metformina, tiazolidinediona ou sulfonilureias. Os estudos evidenciam a possibilidade do uso de tripla terapia com metformina e um dos dois outros fármacos antidiabéticos referidos e podendo utilizar também a insulina, embora com esta última, a evidência da associação seja muito ínfera. ${ }^{8}$

Além da sitagliptina e da vildagliptina, os outros inibidores da DPP-4 em uso comercial, são a saxagliptina, a alogliptina e a linagliptina. As várias medicações diferem entre si principalmente pelo seu estado farmacocinético e pelo seu modo de excreção. A sitagliptina e a alogliptina inibem a DPP-4 através do mecanismo de competição enzimática, já a saxagliptina e a vildagliptina são bloqueadores enzimáticos. A linagliptina é prevalentemente eliminada no fígado pela via hepática, já a saxagliptina é essencialmente eliminada pelos rins pela via renal. Este ponto é interessante, pois a habilidade dos inibidores da DPP-4 atribuírem renoproteção pode divergir, de acordo com a forma de sua eliminação. A linagliptina é o único medicamento que não precisa de ajuste em pacientes com insuficiência renal, não importando o estágio de gravidade que ele apresenta. ${ }^{12}$

Os inibidores da DPP-4 foram bem aceitos em 
estudos de curto prazo, foram semelhantes às descritas com o placebo. Não há nenhum efeito sobre o peso corporal ou o risco de hipoglicemia na ausência de tratamento em conjunto com a insulina ou sulfonilureias. As 3 reações adversas mais relatadas pelos pacientes foram nasofaringite, infecções do trato respiratório superior e cefaleia. A segurança a longo prazo com o inibidor de DPP-4 não foi estabelecida. ${ }^{8},{ }^{12}$

Uma análise de estudo comparando sitagliptina e vildagliptina com dados de efeitos colaterais disponíveis apresentou um mínimo aumento do risco de nasofaringite. Uma outra análise também mostrou um ínfero aumento do risco de nasofaringite em comparação com placebo, que foi principalmente dado pelo subgrupo sitagliptina. O risco de infecções do trato respiratório e urinário superior não foi significativamente aumentado, mostrando um resultado positivo, entretanto o risco de tonturas e dor de cabeça foi ligeiramente aumentado. Pancreatite aguda pode ter um aumento de incidência com os inibidores da DPP-4, embora ainda não existam informações suficientes para saber se existe essa relação e o seu motivo. Se pancreatite for diagnosticada, um inibidor da DPP-4 não é recomendo ser utilizado. Além disso, os inibidores de DPP-4 não devem ser utilizados em doentes com uma história de pancreatite. ${ }^{8}$

Em especial, a linagliptina pode ser considerada uma boa escolha para se ter início na terapia de uma pessoa que tenha doença renal crônica em risco de hipoglicemia ou em um paciente com risco aumentado de desenvolver hipoglicemia. Em alguns estudos, os inibidores da DPP-4 mostraram ser bastante eficientes em pacientes que apresentam doença renal crônica. ${ }^{8}$

Sobre os efeitos gastrointestinais, os inibidores da DPP-4 são medicamentos seguros. Em estudos, os efeitos não desejados que mais se destacaram foram náuseas, vómitos e diarreia, mas todos eles em uma porcentagem muito pequena, quase irrelevante. ${ }^{8},{ }^{12}$

A segurança cardiovascular é um fator de extrema importância para a utilização de novos medicamentos que são comercializados. Seguindo esse parâmetro, alguns estudos que analisam a função cardíaca de doentes, mostraram que fazendo uso de terapias com inibidores da DPP-4 não aparentam indicar qualquer aumento do risco cardiovascular. Entretanto, um número pequeno de pacientes relataram alterações da pressão arterial e a grande maioria que foi realizada esses estudos mostraram não haver nenhum efeito clínico nos sinais vitais, mas ainda é necessário estudos a longo prazo para poder confirmar essa situação descrita. ${ }^{12}$

No que se refere a situações de pacientes que apresentam insuficiência hepática, os inibidores da DPP-4 aparentam ser bem tolerados quando o grau da doença é leve a moderada, o que nos mostra não precisar de nenhum tipo de ajuste de dose em pacientes com essa doença. Entretanto existe uma exceção, para o uso da vildagliptina: ele não é recomendado em doentes com insuficiência hepática que fazem tratamentos com alanina aminotransferase ou aspartato aminotransferase, pois esta associação pode elevar as transferases hepáticas. Para pacientes com insuficiência hepática grave, as informações sobre os efeitos ainda são limitadas e não é recomendado seu uso. ${ }^{12}$

\section{Inibidores dos SGLT-2}

Os SGLT-2 são proteínas presentes no túbulo contornado proximal com a função de realizar o cotransporte de glicose e sódio.

Os inibidores dos cotransportadores de sódioglicose tipo 2 (SGLT-2) são antidiabéticos orais de segunda linha que reduzem a reabsorção de glicose e sódio no túbulo contornado proximal dos rins, causando um aumento da excreção urinária de glicose e sódio, sendo o SGLT-2 responsável pela absorção de $90 \%$ da glicose no rim, enquanto o SGLT1 que está no túbulo contornado distal e realiza a absorção da glicose que restou, sendo responsável por apenas $10 \% .^{13}$ Os inibidores de SGLT-2 possuem um bom perfil de segurança e foram aprovados como monoterapia ou como terapia adjuvante para diabetes mellitus tipo 2. Os mais usados são: dapagliflozina, canagliflozina e empagliflozina. ${ }^{14}$

Dapagliflozina é um inibidor de SGLT-2 muito potente, reversível e seletivo, de administração oral e possui rápida absorção com pico de concentração plasmática em duas horas.15 Sua meia vida dura entre 10 a 17 horas e possui uma ligação à proteínas plasmáticas de $98 \%$, com sua excreção renal baixa. ${ }^{14},{ }^{16}$

Em relação aos seus benefícios, foram comprovados que podem diminuir a hemoglobina glicada ( $\mathrm{HbA} 1 \mathrm{c})$ e reduzir o peso corporal devido à perda calórica de glicosúria, sem aumentar o risco de 
hipoglicemia nos pacientes com DM2. ${ }^{15}$ Reduzem a hipertensão por diminuírem o volume circulante em circunstâncias das suas propriedades diuréticas. Estudos revelam há redução nos níveis de LDL no sangue, diminuindo os riscos de aterosclerose e protegendo o sistema cardiovascular. A dapagliflozina também diminui as chances de desenvolvimento da doença renal, causando a vasodilatação da arteríola eferente e reduzindo a taxa de filtração glomerular o que irá reduzir a progressão da nefropatia diabética. ${ }^{13},{ }^{16}$

A dapagliflozina quando associada a insulina pode causar hipoglicemia. Outros efeitos adversos também foram relatados como infecções genitais, que são rápidas com fácil tratamento. Os efeitos adversos renais foram a insuficiência renal e a redução da depuração de creatinina renal. ${ }^{16}$

A canagliflozina é um inibidor de maior potência, com $99 \%$ ligada a proteínas plasmáticas e pico de concentração plasmática em duas horas. Sua excreção é por meio da via renal, porém sua maioria ocorre pelas fezes. ${ }^{17}$

Em monoterapia ou associadas a outros antidiabéticos, é capaz de reduzir a glicemia e diminuir o peso corporal e pressão arterial nos pacientes com diabetes mellitus tipo 2 . Seus efeitos adversos incluem hipotensão e síncope. A canagliflozina também causa infecções genitais, principalmente nas mulheres, e foi relacionada ao aumento de amputação com seu uso, necessitando de critérios mais rigorosos para seu uso. ${ }^{14} 18$

A empagliflozina possui picos de concentrações plasmáticas em uma hora e meia, sendo rapidamente absorvido e sua excreção é feita por via renal.17 Associada a metformina, a empagliflozina possui efeitos benéficos na redução da hemoglobina glicada (HbA1c), na redução de peso e da pressão arterial. Muitos estudos mostram o efeito benéfico da empagliflozina em relação a rigidez arterial, resistência vascular, adiposidade visceral, do colesterol LDL e HDL. Quando usada como monoterapia, hipoglicemia não é um fator agravante, porém quando usada em associação com sulfonilureias ou insulina, há maior ocorrência de hipoglicemia. Os efeitos adversos mais evidentes infecções micóticas renais devido ao aumento da excreção de glicose. ${ }^{18}$

\section{CONSIDERAÇÕES FINAIS}

Sabendo que o diabetes mellitus é um problema de saúde pública em todo o mundo e que apresenta muitas complicações, podendo levar a óbito, é de suma importância o estudo sobre a mesma e de novas terapias para proporcionar uma melhor qualidade de vida aos pacientes, visto que a maioria deles apresenta outras doenças crônicas, como a hipertensão. Através do estudo desse artigo, podemos perceber que as novas formas de terapia para o tratamento da diabetes mellitus tipo 2, que são os agonistas dos receptores da GLP-1, inibidores da DPP-4 e inibidores da SGLT-2 além de trazerem resultados satisfatórios para o controle da glicemia, também são vistos outros benefícios, como a perda de peso e proteção cardiorrenal em alguns desses fármacos.

Esses medicamentos podem ser utilizados em monoterapia ou associados a outros medicamentos hipoglicemiantes. No caso dos agonistas dos receptores da GLP-1 e dos inibidores da SGLT-2, ao serem utilizados na terapia combinada podem reduzir as complicações cardiorrenais. ${ }^{10}$

Por fim, ressaltamos a importância da mudança do estilo de vida, incluindo a prática de atividade física, mudanças nos hábitos alimentares, além da adesão ao tratamento medicamentoso para que os danos causados pela descompensação da doença não existam ou sejam minimizados.

\section{REFERÊNCIAS}

1 ALVES DA CONCEIÇÃO, R., NOGUEIRA DA SILVA, P., BARBOSA, M. L. C. Fármacos para o Tratamento do Diabetes Tipo II: Uma Visita ao Passado e um Olhar para o Futuro. Rev. Virtual Quim. 2017, 9(2), 514-534.

Disponível em:

<https://www.researchgate.net/profile/Raissa_Alves_Da_Conceicao/publication/316501780_Drugs_for_the_Tr eatment_of_Type_II_Diabetes_A_Visit_to_the_Past_and_a_Look_to_the_Future/links $/ 5 \mathrm{c009e} 5 \mathrm{fa} 6 \mathrm{fdcc} 1 \mathrm{~b} 8 \mathrm{~d} 4 \mathrm{a}$ 9412/Drugs-for-the-Treatment-of-Type-II-Diabetes-A-Visit-to-the-Past-and-a-Look-to-the-Future.pdf>. Acesso em 20 jan 2021.

2 SANTOS, I. C. R. V., CARVALHO, E. F., SOUZA, W. V., MEDEIROS, M. C. W. C. NÓBREGA, N. G. L., LIMA, P. M. S. Complicações crônicas dos diabéticos tipo 2 atendidos nas unidades de saúde da família, Recife, Pernambuco, 
Brasil. Disponível em: < http://www.scielo.br/scielo.php?script=sci_arttext\&pid=S1519-38292008000400008>. Acesso em: 20 jan 2021.

3 BERTONHI, L. G., DIAS, J. C. R. Diabetes mellitus tipo 2: aspectos clínicos, tratamento e conduta dietoterápica. Disponível em:

<http://unifafibe.com.br/revistasonline/arquivos/cienciasnutricionaisonline/sumario/62/18042018212025.pdf >. Acesso em: 18 jan 2021.

4 REYES SANAME, Félix Andrés et al. Tratamiento actual de la diabetes mellitus tipo 2. ccm, Holguín, v. 20, n. 1, p. 98-121, março 2016. Disponível em: <http://scielo.sld.cu/scielo.php?script=sci_arttext\&pid=S156043812016000100009\&Ing=es\&nrm=iso>. Acesso em: 05 fev. 2021.

5 Sociedade Brasileira de Diabetes. Diretrizes da Sociedade Brasileira de Diabetes 2019-2020. Clannad Ed. Cient. 2019; pp. 245-247.

6 LIMA, Ana Filipi Leal Rodrigues. O papel dos inibidores da DPP-4 no tratamento da diabetes do tipo II: uso terapêutico e perspectivas futuras. Universidade Fernando Pessoa. Disponível em <https://bdigital.ufp.pt/bitstream/10284/3204/3/TG_15385.pdf>, acessado em 09 de fevereiro 2021.

7 ARDERIUS, Miguel et al. Liragutido: Avaliação do Benefício Clínico numa População Portuguesa com Diabetes de Difícil Controlo. Rev. Soc. Port. De Medicina Interna, v. 23, n. 4, p. 16-21, out/dez 2016. Disponível em: <http://www.scielo.mec.pt/pdf/mint/v23n4/v23n4a05.pdf>. Acesso em: 18 jan 2021.

8 Neto, Rodrigo Antonio Brandão. Inibidores da DPP-4. MedicinaNet. Disponível em < http://www.medicinanet.com.br/conteudos/revisoes/6948/inibodores_da_dpp_iv.htm>, acesso em 09 de fevereiro de 2021.

9 CARVALHO, D. et al. Agonistas dos receptores do GLP-1 no Tratamento da Diabetes Tipo 2. Rev. Port. De Diabetes; 11 (4); 154-166, dezembro 2016. Disponível em: <https://www.researchgate.net/profile/Jose_SilvaNunes/publication/313315544_Agonistas_dos_Receptores_do_GLP-

1_no_Tratamento_da_Diabetes_Tipo_2_GLP-

1_Receptor_Agonists_in_Type_2_Diabetes_Treatment/links/5895c77ea6fdcc32dbd756e3/Agonistas-dosReceptores-do-GLP-1-no-Tratamento-da-Diabetes-Tipo-2-GLP-1-Receptor-Agonists-in-Type-2-DiabetesTreatment.pdf>. Acesso em: 29 jan 2021.

10 SRIDHAR, Vikas S. et al. Argumentando a favor do uso combinado de inibidores de SGLT2 e agonistas do receptor GLP1 para proteção cardiorrenal. Braz. J. Nephrol, São Paulo , v. 42, n. 4, p. 467-477, Dec. 2020 . Available from <http://www.scielo.br/scielo.php?script=sci_arttext\&pid=S0101-

$28002020000400467 \&$ Ing=en\&nrm=iso>. access on 29 Jan. 2021.

11R. Duarte. Inibidores da DPP-4 (Glipitinas) - 10 anos depois (2007-2017). Revista Portuguesa de Diabetes, 2017, p. 62-67. Disponível em: <http://www.revportdiabetes.com/wp-content/uploads/2017/11/RPD-Vol-12n\%C2\%BA-2-Junho-2017-Artigo-de-Opini\%A3o-p\%C3\%A1gs-62-67.pdf>. Acesso em 09 fev. 2021.

12Reis, Marina Sofia Rodrigues. Inibidores da DPP4: Uma esperança na Nefropatia Diabética?. Faculdade de Medicina da Universidade de Coimbra. Disponível em:

$<$ https://estudogeral.uc.pt/bitstream/10316/30640/1/Inibidores\%20DPP-

4\%20Uma\%20esperan\%c3\%a7a\%20na\%20nefropatia\%20diab\%c3\%a9tica.\%20Marina\%20Reis.\%20FMUC\%202 015.pdf>. Acesso em 09 fev. 2021.

13 Nunes, C. P., Giusti, C. T. Eficácia e Efeitos Adversos dos Inibidores de SGLT-2. Disponível em:

file://C:/Users/andre/Downloads/2231-8498-1-PB.pdf. Acesso em 06 fev 2021.

14 Duarte, R., Araújo, F., Medina, J. L. Perfil Clínico da Dapaglifozina: Um Novo Tratamento da Diabetes Tipo 2. Disponível em file://C:/Users/andre/Downloads/RPD-Setembro-2018-Artigo-de-Revisa\%CC\%83opa\%CC\%81gs-119-132.pdf. Acesso em 11 fev 2021.

15 Dhillon, S. Dapagliflozin: Uma Revisão no Diabetes Tipo 2. Disponível em https://link.springer.com/article/10.1007/s40265-019-01148-3. Acesso em 07 fev 2021.

16 Cada, D. J., Levien, T. L. Dapagliflozin. Disponível em

https://www.ncbi.nlm.nih.gov/pmc/articles/PMC4252236/. Acesso em 07 fev 2021.

17 Dionísio, L. M. C. Inibidores do co-transportador renal de sódio-glicose 2: uma nova classe terapêutica para 
o controlo de glicemia na diabetes. Disponível em https://bdigital.ufp.pt/bitstream/10284/5174/1/PPG_27205.pdf. Acesso em 11 fev 2021.

18 Guthrie R. Canagliflozin and cardiovascular and renal events in type 2 diabetes. Postgrad Med. 2018;130(2):149-153. doi:10.1080/00325481.2018.1423852

19 Nunes, J. S., Aguiar, C., Birne, R., Branco, P., Calado. J., Matos, P., Mello, M., Monteiro, P., Polónia, J.

Empagliflozina e a Nova Era no Tratamento da Diabetes Tipo 2: Para Além do Controlo Glicémico. Disponível em file://C:/Users/andre/Downloads/RPD-Vol-12-n\%C2\%BA-1-Marc\%CC\%A7o-2017-Artigo-de-Revisa\%CC\%83opa\%CC\%81gs-9-22\%20(1).pdf. Acesso em 11 fev 2021. 